Fixed Point Theory, 21(2020), No. 2, 805-818

DOI: $10.24193 /$ fpt-ro.2020.2.57

http://www.math.ubbcluj.ro/ nodeacj/sfptcj.html

\title{
PROPERTIES AND ITERATIVE METHODS FOR THE ELASTIC NET WITH $\ell_{p}$-NORM ERRORS
}

\author{
LILING WEI* AND HONG-KUN XU** \\ *School of Science, Hangzhou Dianzi University, Hangzhou, 310018, China \\ E-mail: wll.1225@foxmail.com \\ ** School of Science, Hangzhou Dianzi University, Hangzhou, 310018, China \\ E-mail: xuhk@hdu.edu.cn (Corresponding author)
}

\begin{abstract}
The $p$-elastic net ( $p$-EN) with $1<p<\infty$ is introduced to recover a sparse signal $x \in \mathbb{R}^{n}$ from $m(<n)$ linear measurements with noise. The $p$-EN, which extends the elastic net of Zou and Hastie [23] and was implicitly suggested by Tropp [16], amounts to minimizing the objective function $(1 / p)\|A x-b\|_{p}^{p}+\lambda\|x\|_{1}+(\mu / 2)\|x\|_{2}^{2}$ over $x \in \mathbb{R}^{n}$, where $A$ is the measurement matrix, $b$ is the observation, and $\lambda>0, \mu>0$ are regularization parameters. Some basic geometric properties of the $p$-EN such as how the solution curve of the minimization depends on the parameters $\lambda$ and $\mu$ are investigated. Moreover, iterative algorithms such as the proximal-gradient algorithm and the Frank-Wolfe algorithm are studied for solving the $p$-EN.
\end{abstract}

Key Words and Phrases: Lasso, compressed sensing, elastic net, $\ell_{p}$-norm error, proximal gradient, Frank-Wolfe.

2010 Mathematics Subject Classification: 49J20, 47J06, 47J25, 47H10, 49N45.

Acknowledgments. The authors were grateful to the anonymous referee for her $/$ his helpful comments and suggestions which improved the presentation of this article. HKX was supported in part by NSFC project number U1811461 and ARC/Discoery project DP200100124.

\section{REFERENCES}

[1] N. Altwaijry, S. Chebbi, H.K. Xu, Properties and splitting methods for the p-elastic net, Pacific J. Optim., 12(2016), no. 4, 801-811.

[2] K. Bredies, D.A. Lorenz, P. Maass, A generalized conditional gradient method and its connection to an iterative shrinkage method, Comput. Optim. Appl., 42(2009), 173-193.

[3] E.J. Candés, The restricted isometry property and its implications for compressed sensing, C.R. Acad. Sci. I, 346(2008), 589-592.

[4] E.J. Candés, J. Romberg, T. Tao, Robust uncertainty principles: Exact signal reconstruction from highly incomplete frequency information, IEEE Trans. Inform. Theory, 52(2006), no. 2, 489-509.

[5] E.J. Candés, J. Romberg, T. Tao, Stable signal recovery from incomplete and inaccurate measurements, Comm. Pure Applied Math., LIX(2006), 1207-1223.

[6] E.J. Candés, M.B. Wakin, An introduction to compressive sampling, IEEE Signal Processing Magazine, 25(2008), no. 2, 21-30. 
[7] P.L. Combettes, R. Wajs, Signal recovery by proximal forward-backward splitting, Multiscale Model. Simul., 4(2005), no. 4, 1168-1200.

[8] I. Daubechies, M. Defrise, C. De Mol, An iterative thresholding algorithm for linear inverse problems with a sparsity constraint, Comm. Pure Appl. Math., 57(2004), 1413-1457.

[9] D.L. Donoho, Compressed sensing, IEEE Trans. Info. Theory, 52(2006), no. 4, 1289-1306.

[10] D.L. Donoho, M. Elad, On the stability of basis pursuit in the presence of noise, Signal Process., 86(2006), no. 3, 511-532.

[11] M. Frank, P. Wolfe, An algorithm for quadratic programming, Naval Research Logistics, Quarterly, 3(1956), 95-110.

[12] M. Hebiri, S. van de Geer, The smooth-lasso and other $\ell_{1}+\ell_{2}$-penalized methods, Electron. J. Statist., 5(2011), 1184-1226.

[13] J.-J. Moreau, Proprietes des applications "prox", C.R. Acad. Sci. Paris Ser. A Math., 256(1963), 1069-1071.

[14] R.T. Rockafellar, Convex Analysis, Princeton University Press, 1970.

[15] R. Tibshirani, Regression shrinkage and selection via the lasso, J. Royal Statist. Soc. Ser. B, 58(1996), 267-288.

[16] J.A. Tropp, Just relax: Convex programming methods for identifying sparse signals in noise, IEEE Transactions on Information Theory, 52(2006), no. 3, 1030-1051.

[17] J. Wright, Y. Ma, Dense error correction via $\ell_{1}$-minimization, IEEE Transactions on Information Theory, 56(2010), no. 7, 3540-3560.

[18] H.K. Xu, Inequalities in Banach spaces with applications, Nonlinear Anal., 16(1991), no. 12, 1127-1138.

[19] H.K. Xu, Properties and iterative methods for the lasso and its variants, Chin. Ann. Math. Ser. B, 35(2014), no. 3, 501-518.

[20] H.K. Xu, Convergence analysis of the Frank-Wolfe algorithm and its generalization in Banach spaces, arXiv2043381.

[21] H.K. Xu, M.A. Alghamdi, N. Shahzad, Regularization for the split feasibility problem, J. Nonlinear Convex Anal., 17(2016), no. 3, 513-525.

[22] M. Yuan, Y. Lin, Model selection and estimation in regression with grouped variables, J. Royal Statist. Soc. Ser. B, 68(2006), 49-67.

[23] H. Zou, T. Hastie, Regularization and variable selection via the elastic net, J. Royal Statist. Soc. Ser. B, 67(2005), 301-320.

Received: September 15, 2019; Accepted: November 10, 2019. 
